\title{
Design of direct acting differential pressure control valve test bench
}

\author{
Ognyan Bekriev ${ }^{1, *}$, and Radoslav Asenov ${ }^{1}$ \\ ${ }^{1}$ Faculty of Power Engineering and Power Machines, "Department of Hydro-aerodynamics and \\ Hydraulic Machines", Technical University of Sofia, 8 St. Kliment Ohridski Blvd, Bulgaria
}

\begin{abstract}
This article aims to acquaint readers with a designed test bench for direct acting differential pressure control valves. These control valves are used in district heating systems. The conformity of the construction of the test bench with the international standard IEC 60534-2-3:2015 is evaluate and the results of an experimental study of the control valve AVPB DN20 Kvs 6.3 PN16 are present.
\end{abstract}

\section{Introduction}

One reason for the widespread use of control valves for differential pressure control, as a component of district heating network substations is the ability to improve the quality of local temperature control of the heat carrier to the internal installations of the users. This is achieved by adjusting the pressure drop on the control valve of the heating circuit and the heat exchanger or by adjusting the pressure drop on the control valve individually. The control valve authority factor "a", is generally defined as the ratio of the pressure drop across the fully open control valve "dPv" and pressure drop across whole circulation circuit "dPc" (Fig.1). In order to achieve quality control of the temperature in the heating system or in the domestic hot water system, it is necessary, the control valve authority factors of the selected control valve to be in the range from 0.5 to 1 . This parameter is related to the deformations of the flow characteristic of the control valves, and thus to the ability of the electronic controllers in the substations to maintain the temperatures set point values, without significant deviations. The differential pressure control valve is also used for balancing the district heating network. This in turn leads to a reduction in the CHP power plant fuel consumption by decreasing the return temperature of the district heating network, and increasing the energy efficiency of the CHP power plant.

where:

$$
a=\frac{\text { differential pressure across the valve }}{\text { differential pressure across the circuit }}=\frac{\mathrm{dPv}}{\mathrm{dPc}}
$$

a - control valve authority factor;

$\mathrm{dPv}$ - differential pressure across the control valve Fig.1;

$\mathrm{dPc}$ - differential pressure across the circuit Fig. 1.

*Corresponding author: bekriev@tu-sofia.bg 

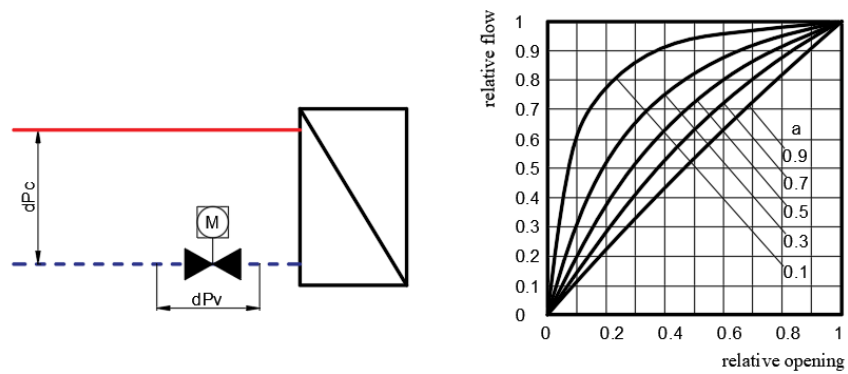

Fig. 1 An example of the influence of the authority factor on the flow characteristic.

In order to ensure a constant differential pressure $(0.8$ bar) for all of the heating circuits in district heating substation it is equipped with a differential pressure controller with a common range from 0.2 to 1 bar. There are a few variants of connection of impulse lines of the differential pressure control valve witch are shown on the next Fig.2.
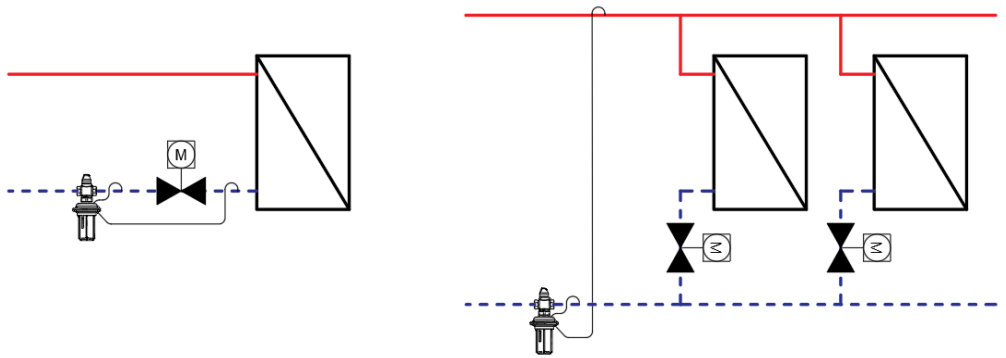

Fig. 2 Differential pressure control valve connection options.

In the central district heating network of Sofia, the pulse lines of the differential pressure control valves are most often connected for control of the differential pressure in two parallel circuits. This stems from the compliance of the design of the district heating substations with the "Basic technical requirements for district heating stations with hot water for the city of Sofia" and "Changes and additions to the basic technical requirements for district heating stations with hot water for the city of Sofia", which contain general instructions for design of the subscriber stations for the district heating network of the city of Sofia.

\section{Construction of the test bench}

The aim of the research is to study the characteristics of differential pressure control valves in the district heating network. The construction of the test bench can recreate a real working conditions. The test bench can be used for studding the operation of the control valves in static and dynamic mode of operation. To achieve a good repeatability of the test results, the construction of the laboratory test bench, have to meet some of the requirements of the international standard IEC 60534-2-3:2015:

- The length of the straight section before the control valve have to be at least $18 \mathrm{x}$ Dn;

- The length of the straight section after the control valve have to be at least $7 \mathrm{x}$ Dn; 
- The flow measurement devices have to have an uncertainty of $\pm 2 \%$ and repeatability $\pm 0.5 \%$

- The accuracy of the temperature measurement device $\mathrm{s}$ be $\pm 1{ }^{0} \mathrm{C}$ or better;

- The device for measuring static pressure before and after the valve with uncertainty of $\pm 2 \%$

- The location and dimensions of the nozzles for outputting a static pressure signal have to be as shown in the standard.

The next figure 3 presents a P\&ID diagram of the laboratory test bench. It consists of the following main elements: buffer vessel, pump (WILO MHI 805-1), throttle valve, solenoid flow meter (PROMAG 33), three-way control valve, manual control valve (HERZ STRÖMAX-GR), differential pressure controller (AVPB DN20 Kvs6.3 PN16), automatic filling valve, safety valve, main expansion vessel, expansion vessel for network storage volume simulation, PLC controller (VISION V120 HMI), pressure transmitter, differential pressure transmitter (E+H PMP 70), electronic micrometric depth gauge (HBM 0..25.4mm \pm $0.01 \mathrm{~mm}$ ), electrical panel (not shown on Fig.3).

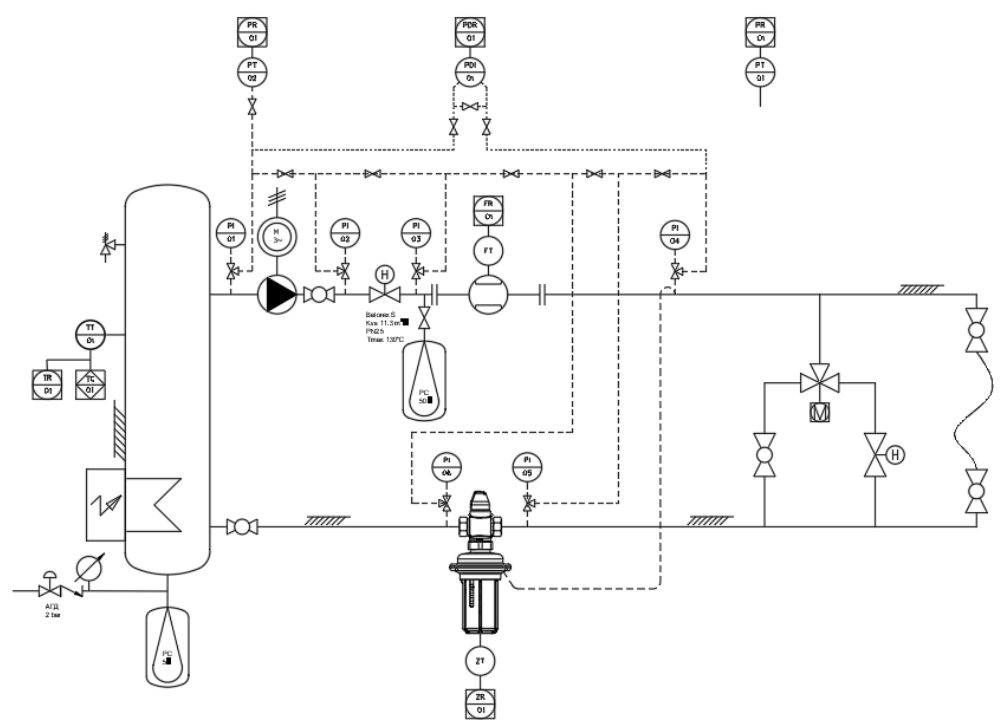

Fig. 3 P\&ID diagram.

\section{Measuring instruments}

The flow rate is measured with a magnetic inductive flow meter PROMAG 33 produced by $\mathrm{E}+\mathrm{H}$. The flow meter is selected to give a maximum accuracy at low flow rates, in accordance with the operating range of the studied differential presure control valve. The flowmeter upstream and downstream straight pipe runs are in accordance with the manufacturer's requirements. The measurement accuracy is $\pm 0.5 \%$ and meets the requirements of the standard. The measuring range is from 0 to $6000 \mathrm{l} / \mathrm{h}$.

The test bench is equipped with a pressure transmitter PMP70 produced by $\mathrm{E}+\mathrm{H}$. By switching the pulse lines, the operator can measure the static pressure at any point of the system. It allows pressure measurements with an accuracy of $\pm 0.1 \%$, which satisfies the requirements of the standard. 
To ensure repeatability of results and high accuracy of measurements, the test bench is equipped with a precision differential pressure transmitter - PMD75 produced by $\mathrm{E}+\mathrm{H}$. It is mounted on a mounting frame that allows alignment and zero-point adjustment. Pulse lines can be switched quickly and easily with visual control for the presence of air bubbles. The measuring sensor is selected with a range that allows the measurement of any drop in differential pressure in the system, without the risk of overload and damage to the device.

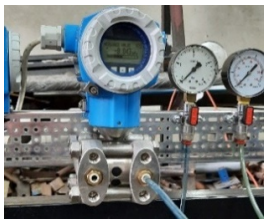

Fig. 4 Differential Pressure Transmitter - Deltabar PMD 75.

The test bench is equipped with a Pt100 type temperature sensor. It is used to measure the temperature of the working fluid and it is connected to fluid temperature control system to a Vision V120 PLC HMI controller. The controller performs the function of a selfadjusting PID controller with pulse width modulation. This allowls the operator to set and control the temperature of the fluid in the system. Additionally, this function the PLC HMI controller is programmed to send an opening and closing signal to the actuator of the threeway control valve, with the possibility of controlling the opening and closing time. With this function the operator can do a dynamic simulations of real operation of the differential pressure control valve.

Two identical Honeywell pressure sensors PG-10 with a time constant of $5 \mathrm{~ms}$, are used to record the pressures before and after the differential pressure control valve during dynamic operation mode simulation. For this purpose, the recording of the rapidly changing pressure signal is performed with a two channel oscilloscope.

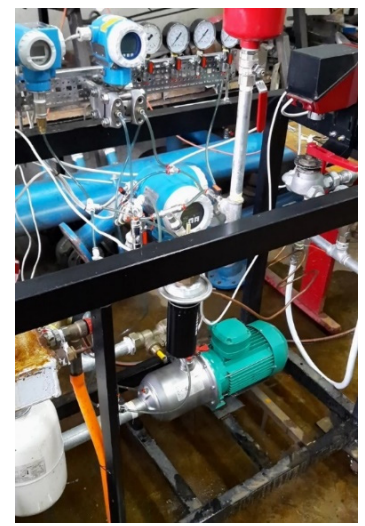

Fig. 5 Image of the test bench.

To ensure safety during operation, the test bench is equipped with an electrical panel, a starter and a fuse for the pump. Power supply units for automatic and control measuring devices are installed in the panel. All terminal blocks are labeled and a preliminary functional test of the power supply of all devices has been performed. 


\section{Results}

Using the test bench described above, several experiments were performed to investigate the characteristics of the AVPB DN20 Kvs 6.3 PN16 differential pressure control valve, Fig.6.
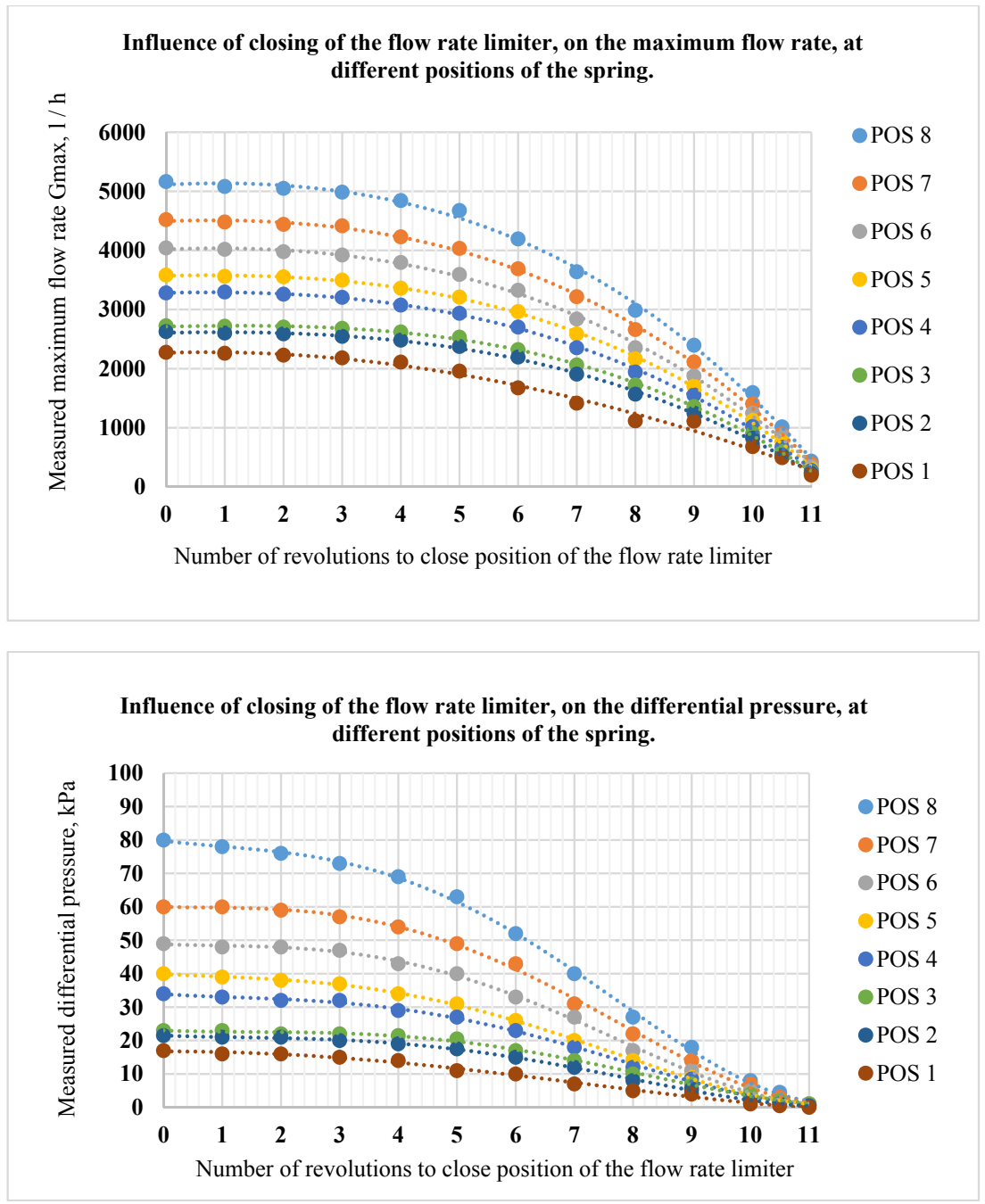

Fig. 6 Results of a study of the operation of the flow rate limiter of the differential pressure control valve AVPB DN20 Kvs 6.3 PN16 produced by Danfoss.

The scale markings are used to establish always the same valve spring tension to adjust the differential pressure. 


\section{Conclusions}

After the initial experiments with a differential pressure control valve AVPB DN20 Kvs 6.3 PN16, the following was achieved:

- The operator has the possibility to adjust the fluid flow in the specified range;

- The operator has the possibility to set and control the static pressure in the system;

- The operator has the possibility to accurately determine the pressure drops by manually switching the pulse lines;

- The operator has the possibility for accurate measurement of static pressure at different points after manual switching;

- The operator has the possibility to set and control the temperature in the system;

- The test bench has the possibility to simulate the characteristics of long heat transfer pipelines, with an efect of elasticity;

- A system for measuring and recording pressure in case of hydraulic shocks has been implemented, which can be simulated by changing the hydraulic stability in the system.

The test bench successfully recreates real working conditions and can be used to study the operation of differential pressure control valves.

\section{References}

1. St. Stamov and collective, Handbook of heat supply and gas supply Part 2, 584, (2000)

2. Al. Kiriy, A. Asenov, Measurement of thermal, hydraulic and mechanical quantities, 260, (2006)

3. Matthias Altendorf and collective, Flow Handbook Endress+Houser,456, (2004)

4. Danfoss, Differential pressure controller with flow limitation (PN 16) AVPB Data sheet 